\title{
The First Answer Set Programming System Competition
}

\author{
Martin Gebser ${ }^{1}$, Lengning Liu $^{2}$, Gayathri Namasivayam ${ }^{2}$, André Neumann ${ }^{1}$, \\ Torsten Schaub ${ }^{\star 1}$, and Mirosław Truszczyński ${ }^{2}$ \\ 1 Institut für Informatik, Universität Potsdam, August-Bebel-Str. 89, D-14482 Potsdam, \\ Germany, \{gebser, aneumann, torsten\}@cs. uni-potsdam. de \\ 2 Department of Computer Science, University of Kentucky, Lexington, KY 40506-0046, USA, \\ \{gayathri,lliul, mirek\}@cs.uky.edu
}

\begin{abstract}
This paper gives a summary of the First Answer Set Programming System Competition that was held in conjunction with the Ninth International Conference on Logic Programming and Nonmonotonic Reasoning. The aims of the competition were twofold: first, to collect challenging benchmark problems, and second, to provide a platform to assess a broad variety of Answer Set Programming systems. The competition was inspired by similar events in neighboring fields, where regular benchmarking has been a major factor behind improvements in the developed systems and their ability to address practical applications.
\end{abstract}

\section{Introduction}

Answer Set Programming (ASP) is an area of knowledge representation concerned with logic-based languages for modeling computational problems in terms of constraints [14]. Its origins are in logic programming $[5,6]$ and nonmonotonic reasoning $[7,8]$. The two areas merged when Gelfond and Lifschitz proposed the answer set semantics for logic programs (also known as the stable model semantics) $[9,10]$. On the one hand, the answer set semantics provided what is now commonly viewed to be the correct treatment of the negation connective in logic programs. On the other hand, with the answer set semantics, logic programming turned out to be a special case of Reiter's default logic [8], with answer sets corresponding to default extensions [11,12].

Answer Set Programming was born when researchers proposed a new paradigm for modeling application domains and problems with logic programs under the answer set semantics: a problem is modeled by a program so that answer sets of the program directly correspond to solutions of the problem $[1,2]$. At about the same time, first software systems to compute answer sets of logic programs were developed: $d l v$ [13] and lparse/smodels [14]. They demonstrated that the answer set programming paradigm has a potential to be the basis for practical declarative computing.

These two software systems, their descendants, and essentially all other ASP systems that have been developed and implemented so far contain two major components. The first of them, a grounder, grounds an input program, that is, produces its propositional equivalent. The second one, a solver, accepts the ground program and actually computes its answer sets (which happen to be the answer sets of the original program).

\footnotetext{
* Affiliated with the School of Computing Science at Simon Fraser University, Burnaby, Canada, and IIIS at Griffith University, Brisbane, Australia.
} 
The emergence of practical software for computing answer sets has been a major impetus behind the rapid growth of ASP in the past decade. Believing that the ultimate success of ASP depends on the continued advances in the performance of ASP software, the organizers of the Ninth International Conference on Logic Programming and Nonmonotonic Reasoning (LPNMR'07) asked us to design and run a contest for ASP software systems. It was more than fitting, given that the first two ASP systems, $d l v$ and lparse/smodels, were introduced exactly a decade ago at the Fourth International Conference on Logic Programming and Nonmonotonic Reasoning (LPNMR'97). We agreed, of course, convinced that as in the case of propositional SATisfiability, where solver competitions have been run for years in conjunction with SAT conferences, this initiative will stimulate research on and development of ASP software, and will bring about dramatic improvements in its capabilities.

In this paper, we report on the project - the First Answer Set Programming System Competition - conducted as part of LPNMR'07. When designing it, we built on our experiences from running preliminary versions of this competition at two Dagstuhl meetings on ASP in 2002 and 2005 [15]. We were also inspired by the approach of and the framework developed for SAT competitions [16], along with the related competitions in solving Quantified Boolean Formulas and Pseudo-Boolean constraints.

The First Answer Set Programming System Competition was run prior to the LPNMR'07 conference. The results are summarized in Section 6 and can be found in full detail at [17]. The competition was run on the Asparagus platform [18], relying on benchmarks stored there before the competition as well as on many new ones submitted by the members of the ASP community (cf. Section 4).

The paper is organized as follows. In the next section, we explain the format of the competition. Section 3 provides a brief overview of the Asparagus platform. In Section 4 and 5, we survey the benchmark problems and the competitors that took part in the competition. The competition results are announced in Section 6. Finally, we discuss our experiences and outline potential future improvements.

\section{Format}

The competition was run in three different categories:

MGS (Modeling, Grounding, Solving) In this category, benchmarks consist of a problem statement, a set of instances (specified in terms of ground facts), and the names of the predicates and their arguments to be used by programmers to encode solutions. The overall performance of software (including both the grounding of input programs and the solving of their ground instantiations) is measured. Success in this category depends on the quality of the input program modeling a problem (the problem encoding), the efficiency of a grounder, and the speed of a solver.

SCore (Solver, Core language) Benchmarks in this category are ground programs in the format common to $d l v$ [19] and lparse [20]. In particular, aggregates are not allowed. Instances are classified further into two subgroups: normal (SCore) and disjunctive $\left(\mathrm{SCore}^{\vee}\right)$. The time needed by solvers to compute answer sets of ground programs is measured. Thus, this category is concerned only with the performance of solvers on ground programs in the basic logic programming syntax. 
SLparse (Solver, Lparse language) Benchmarks in this category are ground programs in the lparse format with aggregates allowed. The performance of solvers on ground programs in lparse format is measured. This category is similar to SCore in that it focuses entirely on solvers. Unlike SCore, though, it assesses the ability of solvers to take advantage of and efficiently process aggregates.

Only decision problems were considered in this first edition of the competition. Thus, every solver had to indicate whether a benchmark instance has an answer set (SAT) or not (UNSAT). Moreover, for instances that are SAT, solvers were required to output a certificate of the existence of an answer set (that is, an answer set itself). This certificate was used to check the correctness of a solution.

The output of solvers had to conform to the following formats (in typewriter font):

SAT: Answer Set: atom1 atom2 ... atomN

The output is one line containing the keywords 'Answer Set:' and the names of the atoms in the answer set. Each atom's name is preceded by a single space. Spaces must not occur within atom names.

UNSAT: No Answer Set

The output is one line containing the keywords 'No Answer Set'.

The competition imposed on each software system (grounder plus solver) the same time and space limits. The number of instances solved within the allocated time and space was used as the primary measure of the performance of a software system. Average running time is only used as a tie breaker.

\section{Platform}

The competition was run on the Asparagus platform [18], providing a web-based benchmarking environment for ASP. The principal goals of Asparagus are (1) to provide an infrastructure for accumulating challenging benchmarks and (2) to facilitate executing ASP software systems under comparable conditions, guaranteeing reproducible and reliable performance results. Asparagus is a continuously running benchmarking environment that combines several internal machines running the benchmarks with an external server for the remaining functionalities including interaction and storage. The internal machines are clones having a modified Linux kernel to guarantee a strict limitation of time and memory resources. This is important in view of controlling heterogeneous ASP solvers that run in multiple processes (e.g., by invoking a stand-alone SAT solver). A more detailed description of the Asparagus platform can be found in [15].

\section{Benchmarks}

The benchmarks for the competition were collected on the Asparagus platform. For all competition categories (MGS, SCore, and SLparse), we asked for submissions of non-ground problem encodings and ground problem instances in separate files.

To add a problem to the MGS category, the author of the problem had to provide 
- a textual problem description that also specified the names and arguments of input and output predicates and

- a set of problem instances in terms of ground facts (using only input predicates).

The submission of a problem encoding was optional for benchmark problems submitted to the MGS category. In most cases, however, the authors provided it, too. In all remaining cases, the competition team provided an encoding. From all benchmark classes already stored on Asparagus or submitted for the competition, we selected several instances for use in the competition (we describe our selection criteria below). For SCore and SLparse, we relied on the availability of encodings to produce ground instances according to the input format of the respective category.

It is important to note that competitors in the MGS category did not have to use the default Asparagus encodings. Instead, they had the option to provide their own problem

\begin{tabular}{|c|c|c|c|}
\hline Benchmark Class & \#Instances & Contributors & $\mathrm{M} \mathrm{C} \mathrm{L}$ \\
\hline 15-Puzzle & $\overline{15}$ & Lengning Liu and Mirosław Truszczyński & $\times-x$ \\
\hline 15-Puzzle & 11 & Asparagus & $-\times-$ \\
\hline Blocked N-Queens & 40 & Gayathri Namasivayam and Mirosław Truszczyński & $\times-x$ \\
\hline Blocked N-Queens & 400 & Asparagus & $-\times-$ \\
\hline Bounded Spanning Tree & 30 & Gayathri Namasivayam and Mirosław Truszczyński & $\times-x$ \\
\hline Car Sequencing & 54 & Marco Cadoli & $\times-x$ \\
\hline Disjunctive Loops & 9 & Marco Maratea & $-\times-$ \\
\hline EqTest & 10 & Asparagus & $-\times-$ \\
\hline Factoring & 10 & Asparagus & $-\times \times$ \\
\hline Fast Food & 221 & Wolfgang Faber & $x-x$ \\
\hline Gebser Suite & 202 & Martin Gebser & $-\times \times$ \\
\hline Grammar-Based Information Extraction & 102 & Marco Manna & $-\times-$ \\
\hline Hamiltonian Cycle & 30 & Lengning Liu and Mirosław Truszczyński & $\times-x$ \\
\hline Hamiltonian Path & 58 & Asparagus & $-\times \times$ \\
\hline Hashiwokakero & 211 & Martin Brain & $--\times$ \\
\hline Hitori & 211 & Martin Brain & $--\times$ \\
\hline Knight's Tour & 165 & Martin Brain & $-x$ \\
\hline Mutex & 7 & Marco Maratea & $-\times-$ \\
\hline Random Non-Tight & 120 & Enno Schultz, Martin Gebser & $-\times \times$ \\
\hline Random Quantified Boolean Formulas & 40 & Marco Maratea & $-\times-$ \\
\hline Reachability & 53 & Giorgio Terracina & $\times \times \times$ \\
\hline RLP & 573 & Yuting Zhao and Fangzhen Lin & $-\times \times$ \\
\hline Schur Numbers & 33 & Lengning Liu and Mirosław Truszczyński & $\times-x$ \\
\hline Schur Numbers & 5 & Asparagus & $-\times-$ \\
\hline Social Golfer & 175 & Marco Cadoli & $x-x$ \\
\hline Sokoban & 131 & Wolfgang Faber & $\times--$ \\
\hline Solitaire Backward & 36 & Martin Brain & $--\times$ \\
\hline Solitaire Backward (2) & 10 & Lengning Liu and Mirosław Truszczyński & $--\times$ \\
\hline Solitaire Forward & 41 & Martin Brain & $--x$ \\
\hline Strategic Companies & 35 & Nicola Leone & $-\times-$ \\
\hline Su-Doku & 8 & Martin Brain & $--x$ \\
\hline TOAST & 54 & Martin Brain & $--\times$ \\
\hline Towers of Hanoi & 29 & Gayathri Namasivayam and Mirosław Truszczyński & $x-x$ \\
\hline Traveling Salesperson & 30 & Lengning Liu and Mirosław Truszczyński & $x-x$ \\
\hline Weight-Bounded Dominating Set & 30 & Lengning Liu and Mirosław Truszczyński & $\times-x$ \\
\hline Weighted Latin Square & 35 & Gayathri Namasivayam and Mirosław Truszczyński & $\times-x$ \\
\hline Weighted Spanning Tree & 30 & Gayathri Namasivayam and Mirosław Truszczyński & $\times-x$ \\
\hline Word Design DNA & 5 & Marco Cadoli & $x-x$ \\
\hline
\end{tabular}

Table 1. Benchmarks submitted by the ASP community. 


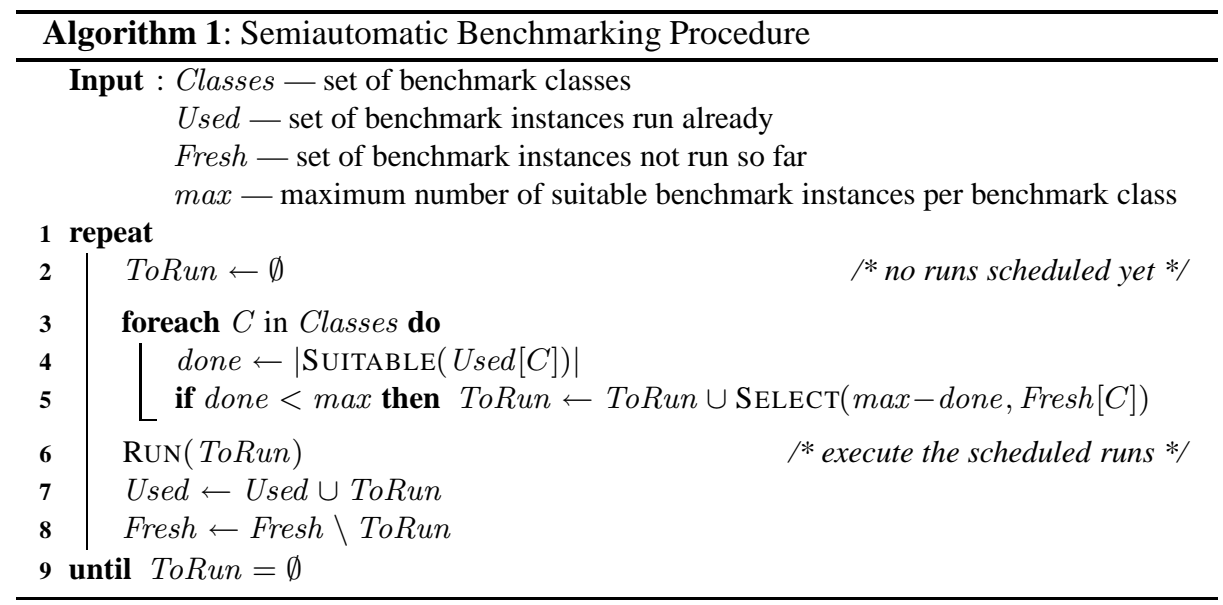

encodings, presumably better than the default ones, as the MGS category was also about assessing the modeling capabilities of grounders and solvers.

The collected benchmarks constitute the result of efforts of the broad ASP community. Table 1 gives an overview of all benchmarks gathered for the competition on the Asparagus platform, listing problems forming benchmark classes, the accompanying number of instances, the names of the contributors, and the associated competition categories (M stands for MGS, C for SCore, and L for SLparse, respectively).

For each competition category, benchmarks were selected by a fixed yet random scheme, shown in Algorithm 1. The available benchmark classes are predefined in Classes. Their instances that have been run already are in Used, the ones not run so far are in Fresh. As an invariant, we impose Used $\cap$ Fresh $=\emptyset$. For a benchmark class $C$ in Classes, we access used and fresh instances via $U s e d[C]$ and Fresh $[C]$, respectively. The maximum number $\max$ of instances per class aims at having approximately 100 benchmark instances overall in the evaluation, that is, $\mid$ Classes $\mid * \max \approx 100$ (if feasible). A benchmark instance in $U$ sed $[C]$ is considered suitable, that is, it is in Suitable $($ Used $[C])$, if at least one call script (see Section 5) was able to solve it and at most three call scripts solved it in less than one second (in other words, some system can solve it, yet it is not too easy). Function $\operatorname{SELECT}(n, \operatorname{Fresh}[C])$ randomly determines $n$ fresh instances from class $C$ if available (if $n \leq|\operatorname{Fresh}[C]|$ ) in order to eventually obtain $\max$ suitable instances of class $C$. Procedure RUN(ToRun) runs all call scripts on the benchmark instances scheduled in ToRun. When ToRun runs empty, no fresh benchmark instances are selectable. If a benchmark class yields less than max suitable instances, Algorithm 1 needs to be re-invoked with an increased max value for the other benchmark classes; thus, Algorithm 1 is "only" semiautomatic. 


\section{Competitors}

As with benchmarks, all executable programs (grounders and solvers) were installed and run on Asparagus. To this end, participants had to obtain Asparagus accounts, unless they already had one, and to register for the respective competition categories.

Different variants of a system were allowed to run in the competition by using different call scripts; per competitor, up to three of them could be registered for each competition category. The list of participating solvers and corresponding call scripts can be found in Table 2 .

\begin{tabular}{|c|c|c|c|c|}
\hline Solver & Affiliation & MGS & SCore & SLparse \\
\hline asper & Angers & & $\begin{array}{l}\text { ASPeR-call-script } \\
\text { ASPeRS20-call-script } \\
\text { ASPeRS30-call-script }\end{array}$ & \\
\hline assat & Hongkong & script.assat.normal & & script.assat.lparse-output \\
\hline clasp & Potsdam & $\begin{array}{l}\text { clasp_cmp_score } \\
\text { clasp_cmp_score } 2 \\
\text { clasp_score_def }\end{array}$ & $\begin{array}{l}\text { clasp_cmp_score_glp } \\
\text { clasp_cmp_score_glp2 } \\
\text { clasp_score_glp_def }\end{array}$ & $\begin{array}{l}\text { clasp_cmp_slparse } \\
\text { clasp_cmp_slparse } 2 \\
\text { clasp_slparse_def }\end{array}$ \\
\hline cmodels & Texas & $\begin{array}{l}\text { default } \\
\text { scriptAtomreasonLp } \\
\text { scriptEloopLp }\end{array}$ & $\begin{array}{l}\text { defaultGlparse.sh } \\
\text { scriptAtomreasonGlparse } \\
\text { scriptEloopGlparse } \\
\text { disjGlparseDefault }^{\vee} \\
\text { disjGparseEloop }^{\vee} \\
\text { disjGparseVerMin }^{\vee}\end{array}$ & $\begin{array}{l}\text { groundedDefault } \\
\text { scriptAtomreasonGr } \\
\text { scriptEloopGr }\end{array}$ \\
\hline dlv & $\begin{array}{l}\text { Vienna/ } \\
\text { Calabria }\end{array}$ & $\begin{array}{l}\text { dlv-contest-special } \\
\text { dlv-contest }\end{array}$ & $\begin{array}{l}\text { dlv-contest-special } \\
\text { dllv-contest }^{\star}\end{array}$ & \\
\hline gnt & Helsinki & \begin{tabular}{|l} 
gnt \\
dencode+gnt \\
dencode_bc+gnt
\end{tabular} & $\begin{array}{l}\text { gnt_score } \\
\text { dencode+gnt_score } \\
\text { dencode_bc+gnt_score }\end{array}$ & $\begin{array}{l}\text { gnt_slparse } \\
\text { dencode+gnt_slparse } \\
\text { dencode_bc+gnt_slparse }\end{array}$ \\
\hline lp2sat & Helsinki & & \begin{tabular}{|l|} 
lp2sat+minisat \\
wf+lp2sat+minisat \\
lp2sat+siege
\end{tabular} & \\
\hline nomore & Potsdam & $\begin{array}{l}\text { nomore-default } \\
\text { nomore-localprop } \\
\text { nomore-D }\end{array}$ & $\begin{array}{l}\text { nomore-default-SCore } \\
\text { nomore-localprop-SCore } \\
\text { nomore-D-SCore }\end{array}$ & $\begin{array}{l}\text { nomore-default-slparse } \\
\text { nomore-localprop-slparse } \\
\text { nomore-D-slparse }\end{array}$ \\
\hline pbmodels & Kentucky & $\begin{array}{l}\text { pbmodels-minisat+-MGS } \\
\text { pbmodels-pueblo-MGS } \\
\text { pbmodels-wsatcc-MGS }\end{array}$ & $\begin{array}{l}\text { pbmodels-minisat+-SCore } \\
\text { pbmodels-pueblo-SCore } \\
\text { pbmodels-wsatcc-SCore }\end{array}$ & $\begin{array}{l}\text { pbmodels-minisat+-SLparse } \\
\text { pbmodels-pueble-SLparse } \\
\text { pbmodels-wsatcc-SLparse }\end{array}$ \\
\hline smodels & Helsinki & $\begin{array}{l}\text { smodels } \\
\text { smodels_rs } \\
\text { smodels_rsn }\end{array}$ & $\begin{array}{l}\text { smodels_score } \\
\text { smodels_rs_score } \\
\text { smodels_rsn_score }\end{array}$ & $\begin{array}{l}\text { smodels_slparse } \\
\text { smodels_rs_slparse } \\
\text { smodels_rsn_slparse }\end{array}$ \\
\hline
\end{tabular}

Table 2. Participating solvers and corresponding call scripts $\left(*^{\star}\right.$ used in both SCore and SCore ${ }^{\vee}$; ${ }^{\vee}$ used in SCore ${ }^{\vee}$ only).

\section{Results}

This section presents the results of the First Answer Set Programming System Competition. The placement of systems was determined according to the number of instances solved within the allocated time and space as the primary measure of performance. Run times were used as tie breakers. Given that each system was allowed to participate in each competition category in three variants, we decided to allocate only one place to 
each system. The place of a system is determined by its best performing variant, represented by a call script.

Each single run was limited to 600 seconds execution time and 448 MB RAM memory usage. For each competition category, we give below tables providing a complete placement of all participating call scripts. We report for each call script the absolute and relative number of solved instances ('Solved' and '\%'), its minimum, maximum, and average run times, where 'avg' gives the average run time on all solved instances and ' $\mathrm{avg}^{\mathrm{t}}$ ' gives the average run time on all instances with a timeout taken as 600 seconds. The last column gives the Euclidean distance between the vector of all run times of a call script and the virtually best solver taken to be the vector of all minimum run times.

For each competition category, we also provide similar data for the used benchmark classes. These tables give the number of instances selected from each class ('\#'), the absolute and relative number of successfully completed runs ('Solved' and ' $\%$ ') aggregated over all instances and call scripts, the same separately for satisfiable ('SAT') and unsatisfiable ('UNSAT') instances, and finally, the minimum, maximum, and average times over all successfully completed runs on the instances of a class.

Finally, we chart the numbers of solved instances and the solving times of participating call scripts for each competition category. See Section 6.1 for an exemplary description of these graphics.

More statistics and details are available at [17].

\subsection{Results of the MGS competition}

\begin{tabular}{|c|l|r|r|r|r|r|r|r|}
\hline Place & \multicolumn{1}{|c|}{ Call Script } & \multicolumn{1}{c|}{ Solved } & \multicolumn{1}{c|}{$\%$} & min & \multicolumn{1}{c|}{$\max$} & \multicolumn{1}{c|}{ avg } & \multicolumn{1}{c|}{ avg $^{t}$} & EuclDist \\
\hline \hline 1 & dlv-contest-special & $76 / 119$ & 63.87 & 0.07 & 565.38 & 54.31 & 251.49 & 3542.79 \\
2 & dlv-contest & $66 / 119$ & 55.46 & 0.06 & 579.09 & 49.73 & 294.81 & 3948.3 \\
3 & pbmodels-minisat+-MGS & $65 / 111$ & 58.56 & 0.52 & 563.39 & 83.27 & 297.41 & 4142.88 \\
4 & clasp_cmp_score2 & $64 / 111$ & 57.66 & 0.87 & 579.14 & 115.35 & 320.56 & 4285.59 \\
5 & clasp_score_def & $60 / 111$ & 54.05 & 0.91 & 542.64 & 80.09 & 318.97 & 4277.69 \\
6 & clasp_cmp_score & $58 / 111$ & 52.25 & 0.83 & 469.46 & 87.40 & 332.16 & 4280.92 \\
7 & pbmodels-pueble-MGS & $54 / 111$ & 48.65 & 0.34 & 584.31 & 80.94 & 347.49 & 4491.85 \\
8 & default & $51 / 119$ & 42.86 & 0.23 & 453.88 & 64.96 & 370.7 & 4543.12 \\
9 & smodels_rs & $34 / 118$ & 28.81 & 0.30 & 539.33 & 153.86 & 471.45 & 5349.11 \\
10 & smodels & $34 / 104$ & 32.69 & 1.14 & 584.06 & 173.60 & 460.6 & 4974.14 \\
11 & pbmodels-wsatcc-MGS & $23 / 111$ & 20.72 & 1.05 & 563.52 & 136.97 & 504.06 & 5409.17 \\
12 & smodels_rsn & $22 / 111$ & 19.82 & 0.12 & 579.10 & 163.14 & 513.42 & 5471.81 \\
13 & nomore-default & $13 / 111$ & 11.71 & 22.04 & 521.11 & 315.78 & 566.71 & 5714.67 \\
14 & scriptAtomreasonLp & $12 / 24$ & 50.00 & 3.59 & 259.88 & 91.18 & 345.59 & 2121.13 \\
15 & nomore-localprop & $10 / 111$ & 9.01 & 19.54 & 521.03 & 324.83 & 575.21 & 5787.71 \\
16 & nomore-D & $9 / 111$ & 8.11 & 48.50 & 473.89 & 161.99 & 564.49 & 5763.96 \\
17 & scriptEloopLp & $4 / 16$ & 25.00 & 49.92 & 223.03 & 106.09 & 476.52 & 2088.98 \\
18 & gnt & $0 / 8$ & 0.00 & & & & 600 & 1696.31 \\
19 & dencode+gnt & $0 / 8$ & 0.00 & & & & 600 & 1696.31 \\
20 & dencode_bc+gnt & $0 / 8$ & 0.00 & & & & 600 & 1696.31 \\
21 & script.assat.normal & $0 / 50$ & 0.00 & & & & 600 & 4101.66 \\
\hline
\end{tabular}

Table 3. Placing of call scripts in the MGS competition.

The winners of the MGS competition are: 


\begin{tabular}{|c|c|c|c|c|c|c|c|c|c|c|}
\hline Benchmark Class & \# & Solved & $\%$ & SAT & $\%$ & UNSAT & $\%$ & $\min$ & $\max$ & avg \\
\hline Sokoban & & $16 / 16$ & 100.00 & $6 / 6$ & 100.00 & $10 / 10$ & 100.00 & 12.66 & 109.52 & 54.39 \\
\hline Weighted Spanning Tree & 8 & $89 / 127$ & 70.08 & $89 / 127$ & 70.08 & $0 / 0$ & & 0.06 & 579.10 & 115.27 \\
\hline Social Golfer & 8 & $75 / 120$ & 62.50 & $59 / 75$ & 78.67 & $16 / 45$ & 35.56 & 0.23 & 579.09 & 40.34 \\
\hline Bounded Spanning Tree & 8 & 73/127 & 57.48 & 73/127 & 57.48 & $0 / 0$ & & 0.23 & 519.09 & 65.47 \\
\hline Towers of Hanoi] & 8 & $71 / 136$ & 52.21 & 71/136 & 52.21 & $0 / 0$ & & 1.74 & 584.31 & 101.10 \\
\hline Blocked N-Queens & 8 & $60 / 120$ & 50.00 & $44 / 75$ & 58.67 & $16 / 45$ & 35.56 & 6.50 & 542.64 & 181.69 \\
\hline Hamiltonian Cycle & 8 & $64 / 150$ & 42.67 & $64 / 150$ & 42.67 & $0 / 0$ & & 0.88 & 453.88 & 57.46 \\
\hline Weighted Latin Square & 8 & $41 / 120$ & 34.17 & $22 / 45$ & 48.89 & $19 / 75$ & 25.33 & 0.12 & 477.67 & 93.04 \\
\hline t-Bounded Dominating Set & 8 & $42 / 127$ & 33.07 & $42 / 127$ & 33.07 & $0 / 0$ & & 0.83 & 563.39 & 127.87 \\
\hline Schur N & & $32 / 120$ & 26.67 & $18 / 90$ & 20.00 & $14 / 30$ & 46.67 & 3.27 & 468.79 & 120.96 \\
\hline 15 & 7 & $24 / 105$ & 22.86 & $24 / 105$ & 22.86 & $0 / 0$ & & 52.91 & 579.14 & 291.20 \\
\hline Car Sequ & 8 & $25 / 120$ & 20.83 & $24 / 105$ & 22.86 & $1 / 15$ & 6.67 & 0.69 & 563.52 & 106.08 \\
\hline Fast & 8 & $19 / 127$ & 14.96 & $8 / 64$ & 12.50 & $11 / 63$ & 17.46 & 5.30 & 352.11 & 113.08 \\
\hline Traveling Salesperson & 8 & $16 / 128$ & 12.50 & $16 / 128$ & 12.50 & $0 / 0$ & & 0.72 & 11.18 & 2.17 \\
\hline Reachability & & $8 / 160$ & 5.00 & $6 / 120$ & 5.00 & $2 / 40$ & 5.00 & 0.26 & 169.90 & 49.48 \\
\hline
\end{tabular}

Table 4. Benchmarks used in the MGS competition.

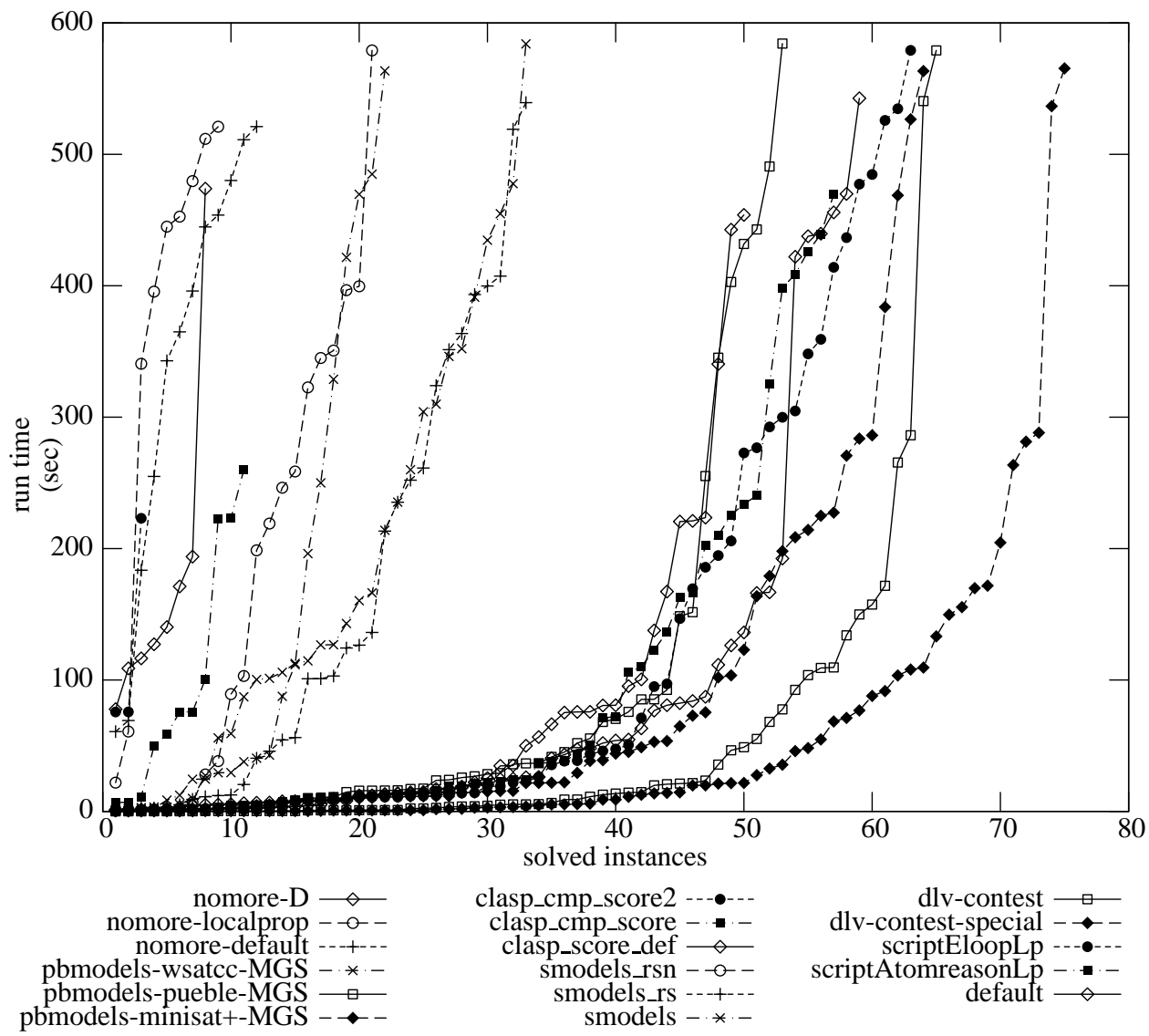

Fig. 1. Chart of the MGS competition. 


$\begin{aligned} \text { FIRST PLACE WINNER } & d l v \\ \text { SECOND PLACE WINNER } & \text { pbmodels } \\ \text { THIRD PLACE WinNER } & \text { clasp }\end{aligned}$

The detailed placement of call scripts is given in Table 3. Table 4 gives statistics about the benchmark classes used in the MGS competition. The performance of all participating call scripts is also given in graphical form in Figure 1. Thereby, the $x$-axis represents the number of (solved) benchmark instances, and the $y$-axis represents time. For a given call script, the solved instances are ordered by run times, and a point $(x, y)$ in the chart expresses that the $x$ th instance was solved in $y$ seconds. The more to the right the curve of a call script ends, the more benchmark instances were solved within the allocated time and space. Since the number of solved instances is our primary measure of performance, the rightmost call script is the winner of the MGS competition.

\subsection{Results of the SCore competition}

\begin{tabular}{|c|c|c|c|c|c|c|c|c|}
\hline Place & Call Script & Solved & $\%$ & $\min$ & $\max$ & avg & $\operatorname{avg}^{t}$ & EuclDist \\
\hline$\overline{1} 1$ & clasp_cmp_score_glp2 & $\begin{array}{ll}89 / 95 \\
\end{array}$ & 93.68 & $\overline{0.56}$ & $\overline{530.21}$ & 29.81 & $\overline{65.82}$ & 1080.46 \\
\hline 2 & clasp_cmp_score_glp & $89 / 95$ & 93.68 & 0.75 & 504.49 & 30.36 & 66.34 & 1099.14 \\
\hline 3 & clasp_score_glp_def & $86 / 95$ & 90.53 & 0.75 & 431.66 & 25.20 & 79.66 & 1386.63 \\
\hline 4 & smodels_rs_score & $81 / 95$ & 85.26 & 1.21 & 346.36 & 38.93 & 121.61 & 1872.81 \\
\hline 5 & defaultGlparse.sh & $81 / 95$ & 85.26 & 1.35 & 597.97 & 46.86 & 128.38 & 2089.18 \\
\hline 6 & scriptAtomreasonGlparse & $80 / 95$ & 84.21 & 1.30 & 576.80 & 42.40 & 130.44 & 2107.83 \\
\hline 7 & pbmodels-minisat+-SCore & $80 / 95$ & 84.21 & 0.72 & 436.11 & 57.18 & 142.89 & 2170.4 \\
\hline 8 & pbmodels-pueblo-SCore & $78 / 95$ & 82.11 & 0.34 & 452.84 & 41.00 & 141.03 & 2210.39 \\
\hline 9 & dencode+gnt_score & $78 / 95$ & 82.11 & 1.27 & 363.19 & 42.80 & 142.51 & 2162.64 \\
\hline 10 & smodels_score & $77 / 95$ & 81.05 & 1.28 & 352.41 & 40.40 & 146.43 & 2217.61 \\
\hline 11 & dencode_bc+gnt_score & $77 / 95$ & 81.05 & 1.27 & 360.70 & 42.52 & 148.15 & 2228.65 \\
\hline 12 & re & $77 / 95$ & 81.05 & 1.27 & 359.77 & 42.56 & 148.18 & 2228.83 \\
\hline 13 & IloopGlparse & $75 / 95$ & 78.95 & 1.36 & 598.20 & 42.86 & 160.15 & 2493.41 \\
\hline 14 & ls_rsn_score & $75 / 95$ & 78.95 & 1.21 & 486.23 & 63.00 & 176.05 & 2503.32 \\
\hline 15 & ninisat & $75 / 95$ & 78.95 & 1.10 & 561.06 & 79.89 & 189.39 & 2621.13 \\
\hline 16 & $\mathrm{wf}+\mathrm{lp} 2 \mathrm{sat}+$ minisat & $73 / 95$ & 76.84 & 1.56 & 587.40 & 86.42 & 205.35 & 2792.51 \\
\hline 17 & dlv-contest-special & $69 / 95$ & 72.63 & 0.24 & 586.62 & 102.47 & 238.64 & 3090.71 \\
\hline 18 & dlv-contest & $68 / 95$ & 71.58 & 0.24 & 587.83 & 96.69 & 239.74 & 3110.36 \\
\hline 19 & lp2sat+siege & $68 / 95$ & 71.58 & 1.11 & 471.36 & 97.50 & 240.32 & 3052.8 \\
\hline 20 & nomore-localprop-SCore & $64 / 95$ & 67.37 & 2.45 & 550.43 & 103.23 & 265.34 & 3316.33 \\
\hline 21 & nomore-default-SCore & $63 / 95$ & 66.32 & 2.45 & 554.76 & 124.62 & 284.75 & 3415.78 \\
\hline 22 & nomore-D-SCore & $62 / 95$ & 65.26 & 2.77 & 559.88 & 161.15 & 313.59 & 3583.85 \\
\hline 23 & ASPeR-call-script & $24 / 95$ & 25.26 & 1.47 & 592.24 & 98.28 & 473.25 & 4906.79 \\
\hline 24 & 11-script & $21 / 95$ & 22.11 & 1.51 & 561.20 & 88.99 & 487.04 & 4995.78 \\
\hline 25 & ASPeRS20-call-script & $21 / 95$ & 22.11 & 1.49 & 381.33 & 89.40 & 487.13 & 4980.24 \\
\hline 26 & pbmodels-wsatcc-SCore & $6 / 95$ & 6.32 & 25.57 & 529.80 & 208.15 & 575.25 & 5514.97 \\
\hline
\end{tabular}

Table 5. Placing of call scripts in the SCore competition.

The winners of the SCore competition are:

$\begin{aligned} \text { First Place Winner } & \text { clasp } \\ \text { SECOND Place Winner } & \text { smodels } \\ \text { ThIRD Place Winner } & \text { cmodels }\end{aligned}$




\begin{tabular}{|r|r|r|c|r|r|r|r|r|r|r|}
\hline Benchmark Class & \multicolumn{1}{|c|}{ Solved } & \multicolumn{1}{c|}{$\%$} & \multicolumn{1}{|c|}{ SAT } & \multicolumn{1}{c|}{$\%$} & UNSAT & $\%$ & min & max & \multicolumn{1}{c|}{ avg } \\
\hline \hline 15-Puzzle & 10 & $236 / 260$ & 90.77 & $121 / 130$ & 93.08 & $115 / 130$ & 88.46 & 0.74 & 480.13 & 25.49 \\
Factoring & 5 & $114 / 130$ & 87.69 & $46 / 52$ & 88.46 & $68 / 78$ & 87.18 & 1.21 & 554.76 & 50.35 \\
RLP-150 & 14 & $306 / 364$ & 84.07 & $21 / 26$ & 80.77 & $285 / 338$ & 84.32 & 0.34 & 205.03 & 22.01 \\
RLP-200 & 14 & $287 / 364$ & 78.85 & $0 / 0$ & & $287 / 364$ & 78.85 & 0.39 & 581.98 & 75.21 \\
Schur Numbers & 5 & $99 / 130$ & 76.15 & $88 / 104$ & 84.62 & $11 / 26$ & 42.31 & 2.76 & 561.20 & 49.82 \\
EqTest & 5 & $93 / 130$ & 71.54 & $0 / 0$ & & $93 / 130$ & 71.54 & 0.66 & 592.24 & 75.02 \\
Hamiltonian Path & 14 & $219 / 364$ & 60.16 & $201 / 338$ & 59.47 & $18 / 26$ & 69.23 & 0.24 & 559.88 & 64.74 \\
Random Non-Tight & 14 & $216 / 364$ & 59.34 & $38 / 52$ & 73.08 & $178 / 312$ & 57.05 & 0.57 & 598.20 & 121.87 \\
Blocked N-Queens & 14 & $167 / 364$ & 45.88 & $55 / 156$ & 35.26 & $112 / 208$ & 53.85 & 13.70 & 587.40 & 110.59 \\
\hline
\end{tabular}

Table 6. Benchmarks used in the SCore competition.

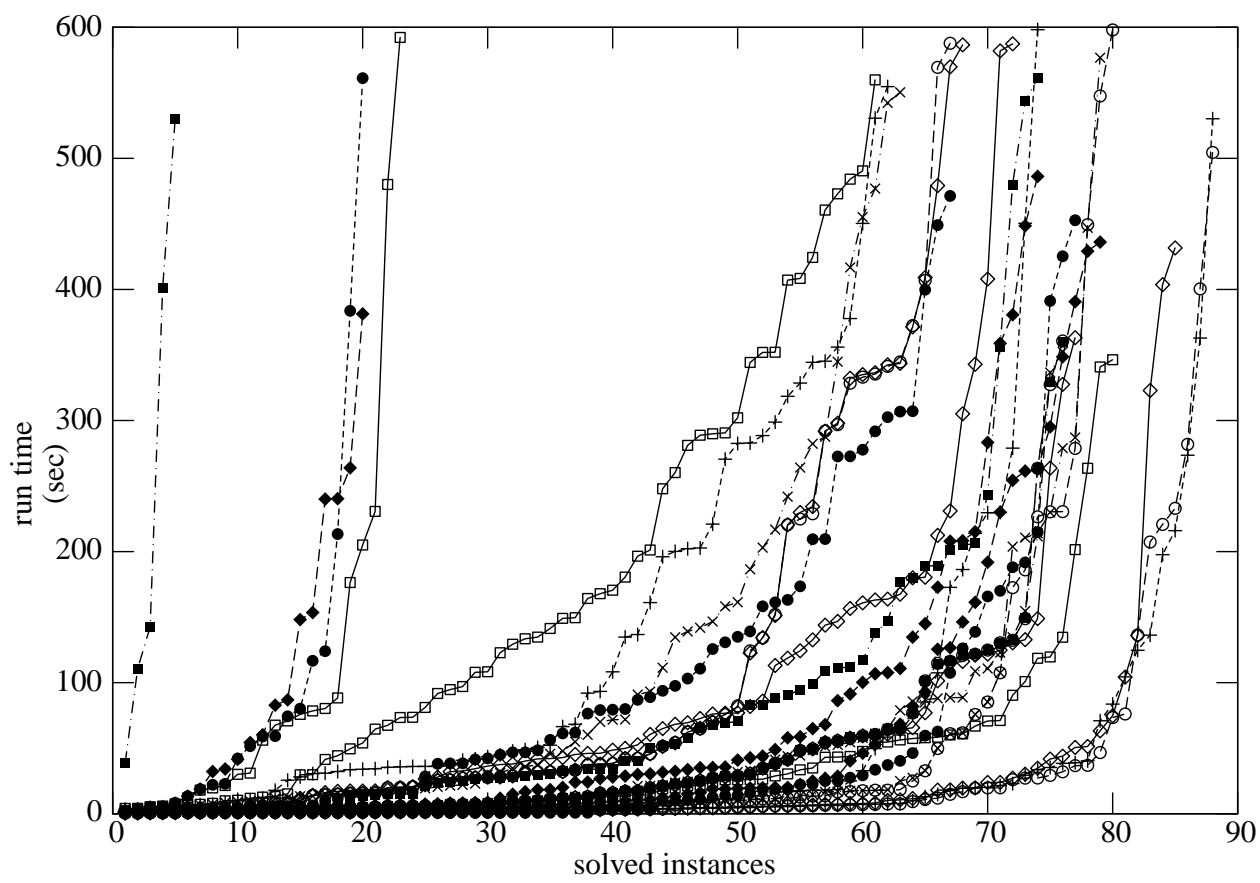

dlv-contest-special $\multimap$ dlv-contest $--\mathrm{o-}-$ nomore-default-SCore ---+--nomore-localprop-SCore $-\cdot * \cdot-$ nomore-D-SCore $-\square$ pbmodels-minisat+-SCore ---pbmodels-pueblo-SCore -- - -

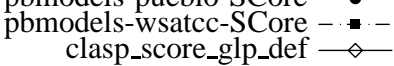

clasp_cmp_score_glp - - - clasp_cmp_score_gIp 2 ---+---scii smodels_score - * *smodels_rsn_score - - - lp2sat+siege --lp2sat+minisat - -

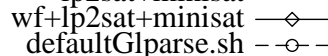

scriptEloopGlparse -..---. iptAtomreasonGlparse - * *ASPeR-call-script ASPeRS20-call-script $-\bullet-$ ASPeRS30-call-script -- $\bullet$ gnt_score - dencode_bc+gnt_score $-\diamond-$

Fig. 2. Chart of the SCore competition.

The detailed placement of call scripts is given in Table 5. Table 6 gives statistics about the benchmark classes used in the SCore competition. The performance of all participating call scripts is charted in Figure 2.

The winners of the $\mathrm{SCore}^{\vee}$ competition are: 


\begin{tabular}{|c|l|r|r|r|r|r|r|r|}
\hline Place & \multicolumn{1}{|c|}{ Call Script } & Solved & \multicolumn{1}{c|}{$\%$} & $\min$ & $\max$ & avg & avg $^{t}$ & EuclDist \\
\hline \hline 1 & dlv-contest-special & $54 / 55$ & 98.18 & 0.03 & 258.73 & 23.59 & 34.07 & 279.35 \\
2 & dlv-contest & $54 / 55$ & 98.18 & 0.03 & 259.97 & 23.86 & 34.33 & 279.44 \\
3 & disjGlparseDefault & $33 / 55$ & 60.00 & 1.06 & 521.59 & 54.49 & 272.69 & 2631.4 \\
4 & dencode+gnt_score & $29 / 55$ & 52.73 & 2.23 & 521.51 & 56.34 & 313.34 & 2922.73 \\
5 & gnt_score & $29 / 55$ & 52.73 & 2.21 & 521.91 & 56.44 & 313.4 & 2922.87 \\
6 & dencode_bc+gnt_score & $29 / 55$ & 52.73 & 2.22 & 522.63 & 56.45 & 313.4 & 2923.17 \\
7 & disjGparseEloop & $27 / 55$ & 49.09 & 1.21 & 521.55 & 33.83 & 322.06 & 2978.46 \\
8 & disjGparseVerMin & $27 / 55$ & 49.09 & 1.22 & 523.40 & 33.98 & 322.14 & 2978.77 \\
\hline
\end{tabular}

Table 7. Placing of call scripts in the SCore ${ }^{\vee}$ competition.

\begin{tabular}{|r|r|r|r|r|r|r|r|r|r|r|}
\hline Benchmark Class & 作 & Solved & \multicolumn{1}{|c|}{$\%$} & \multicolumn{1}{|c|}{ SAT } & \multicolumn{1}{c|}{$\%$} & UNSAT & \multicolumn{1}{c|}{$\%$} & $\min$ & max & avg \\
\hline Grammar-Based Information Extraction & 15 & $120 / 120$ & 100.00 & $64 / 64$ & 100.00 & $56 / 56$ & 100.00 & 0.62 & 7.86 & 5.03 \\
Disjunctive Loops & 3 & $21 / 24$ & 87.50 & $0 / 0$ & & $21 / 24$ & 87.50 & 0.44 & 522.63 & 95.24 \\
Strategic Companies & 15 & $88 / 120$ & 73.33 & $88 / 120$ & 73.33 & $0 / 0$ & & 0.35 & 523.40 & 71.22 \\
Mutex & 7 & $18 / 56$ & 32.14 & $0 / 0$ & & $18 / 56$ & 32.14 & 0.03 & 259.97 & 37.41 \\
Random Quantified Boolean Formulas & 15 & $35 / 120$ & 29.17 & $0 / 0$ & & $35 / 120$ & 29.17 & 0.11 & 290.99 & 44.41 \\
\hline
\end{tabular}

Table 8. Benchmarks used in the SCore ${ }^{\vee}$ competition.

$\begin{aligned} \text { FIRST Place WINNER } & d l v \\ \text { SECOND Place WinNer } & \text { cmodels } \\ \text { THIRD Place WinNer } & \text { gnt }\end{aligned}$

The detailed placement of call scripts is given in Table 7. Table 8 gives statistics about the benchmark classes used in the SCore ${ }^{\vee}$ competition. The performance of all participating call scripts is charted in Figure 3.

\subsection{Results of the SLparse competition}

The winners of the SLparse competition are:

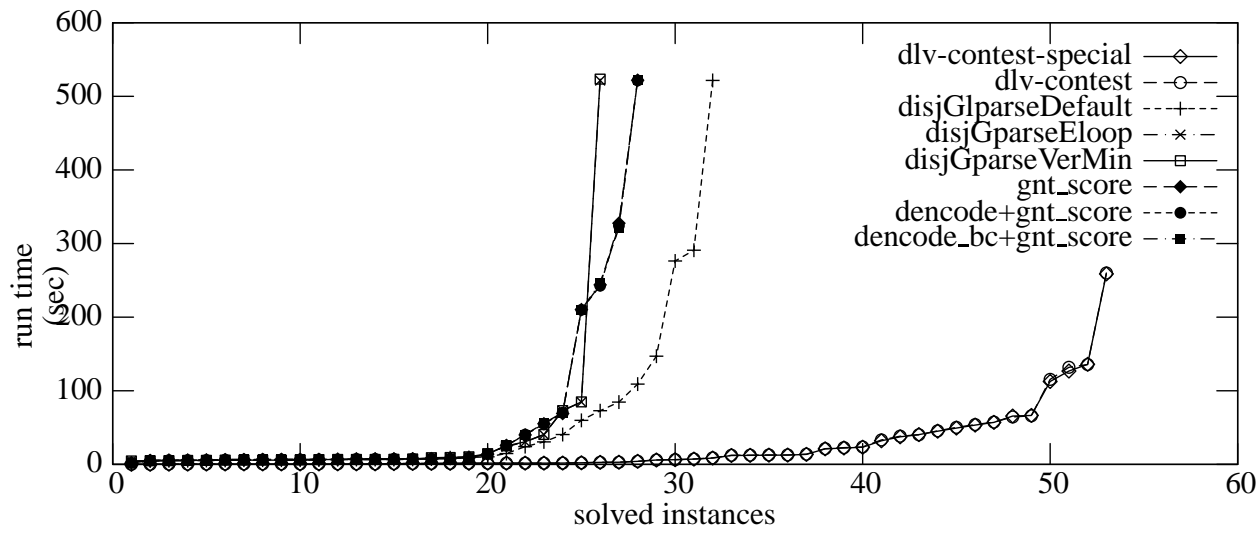

Fig. 3. Chart of the SCore ${ }^{\vee}$ competition. 


\begin{tabular}{|c|l|r|r|r|r|r|r|r|}
\hline Place & \multicolumn{1}{|c|}{ Call Script } & \multicolumn{1}{c|}{ Solved } & \multicolumn{1}{c|}{$\%$} & $\min$ & \multicolumn{1}{c|}{$\max$} & \multicolumn{1}{c|}{ avg } & \multicolumn{1}{|c|}{ avg $^{t}$} & EuclDist \\
\hline \hline 1 & clasp_cmp_slparse2 & $100 / 127$ & 78.74 & 0.38 & 556.49 & 75.96 & 187.37 & 2791.89 \\
2 & llasp_cmp_slparse & $94 / 127$ & 74.02 & 0.41 & 502.53 & 61.37 & 201.33 & 2919.46 \\
3 & pbmodels-minisat+-SLparse & $91 / 127$ & 71.65 & 0.49 & 503.57 & 76.69 & 225.03 & 3241.06 \\
4 & llasp_slparse_def & $89 / 127$ & 70.08 & 0.37 & 546.50 & 55.62 & 218.5 & 3152.34 \\
5 & smodels_rs_slparse & $87 / 127$ & 68.50 & 0.23 & 576.28 & 95.92 & 254.69 & 3403.9 \\
6 & groundedDefault & $81 / 127$ & 63.78 & 0.25 & 407.49 & 46.20 & 246.79 & 3448.34 \\
7 & scriptAtomreasonGr & $81 / 127$ & 63.78 & 0.25 & 407.46 & 50.55 & 249.56 & 3465.67 \\
8 & scriptEloopGr & $78 / 127$ & 61.42 & 0.24 & 407.48 & 46.15 & 259.84 & 3598.7 \\
9 & smodels_slparse & $75 / 127$ & 59.06 & 0.26 & 518.08 & 102.76 & 306.35 & 3958.86 \\
10 & smodels_rsn_slparse & $74 / 127$ & 58.27 & 0.35 & 596.39 & 70.52 & 291.49 & 3815.31 \\
11 & pbmodels-pueblo-SLparse & $69 / 127$ & 54.33 & 0.25 & 593.05 & 87.25 & 321.42 & 4189.03 \\
12 & nomore-D-slparse & $54 / 127$ & 42.52 & 1.08 & 530.39 & 152.78 & 409.84 & 4765.06 \\
13 & nomore-localprop-slparse & $50 / 127$ & 39.37 & 1.08 & 517.63 & 120.80 & 411.34 & 4846.58 \\
14 & nomore-default-slparse & $49 / 127$ & 38.58 & 1.08 & 549.80 & 143.44 & 423.85 & 4920.23 \\
15 & gnt_slparse & $35 / 127$ & 27.56 & 2.10 & 482.13 & 81.40 & 457.08 & 5276.26 \\
16 & dencode+gnt_slparse & $35 / 127$ & 27.56 & 2.18 & 482.81 & 81.64 & 457.15 & 5276.38 \\
17 & dencode_bc+gnt_slparse & $35 / 127$ & 27.56 & 2.10 & 485.36 & 81.70 & 457.16 & 5276.53 \\
18 & script.assat.lparse-output & $30 / 127$ & 23.62 & 1.00 & 225.28 & 38.64 & 467.4 & 5379.18 \\
19 & pbmodels-wsatcc-SLparse & $25 / 127$ & 19.69 & 1.12 & 272.98 & 46.56 & 491.05 & 5585.82 \\
\hline
\end{tabular}

Table 9. Placing of call scripts in the SLparse competition.

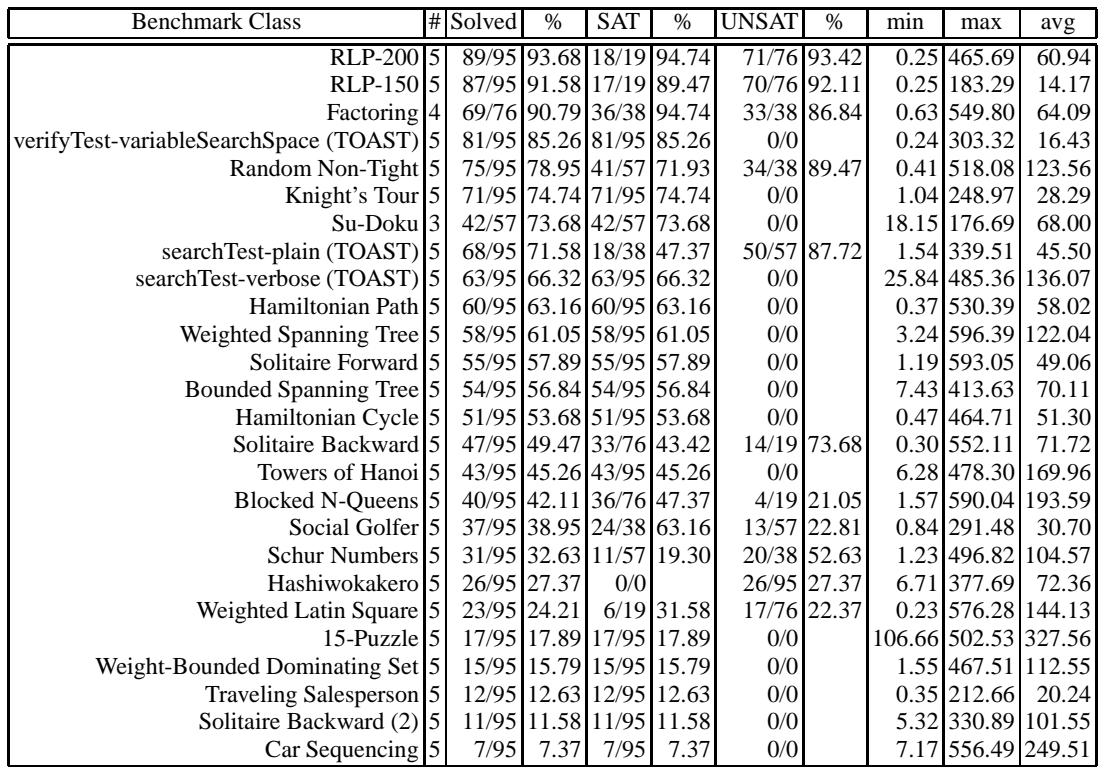

Table 10. Benchmarks used in the SLparse competition. 

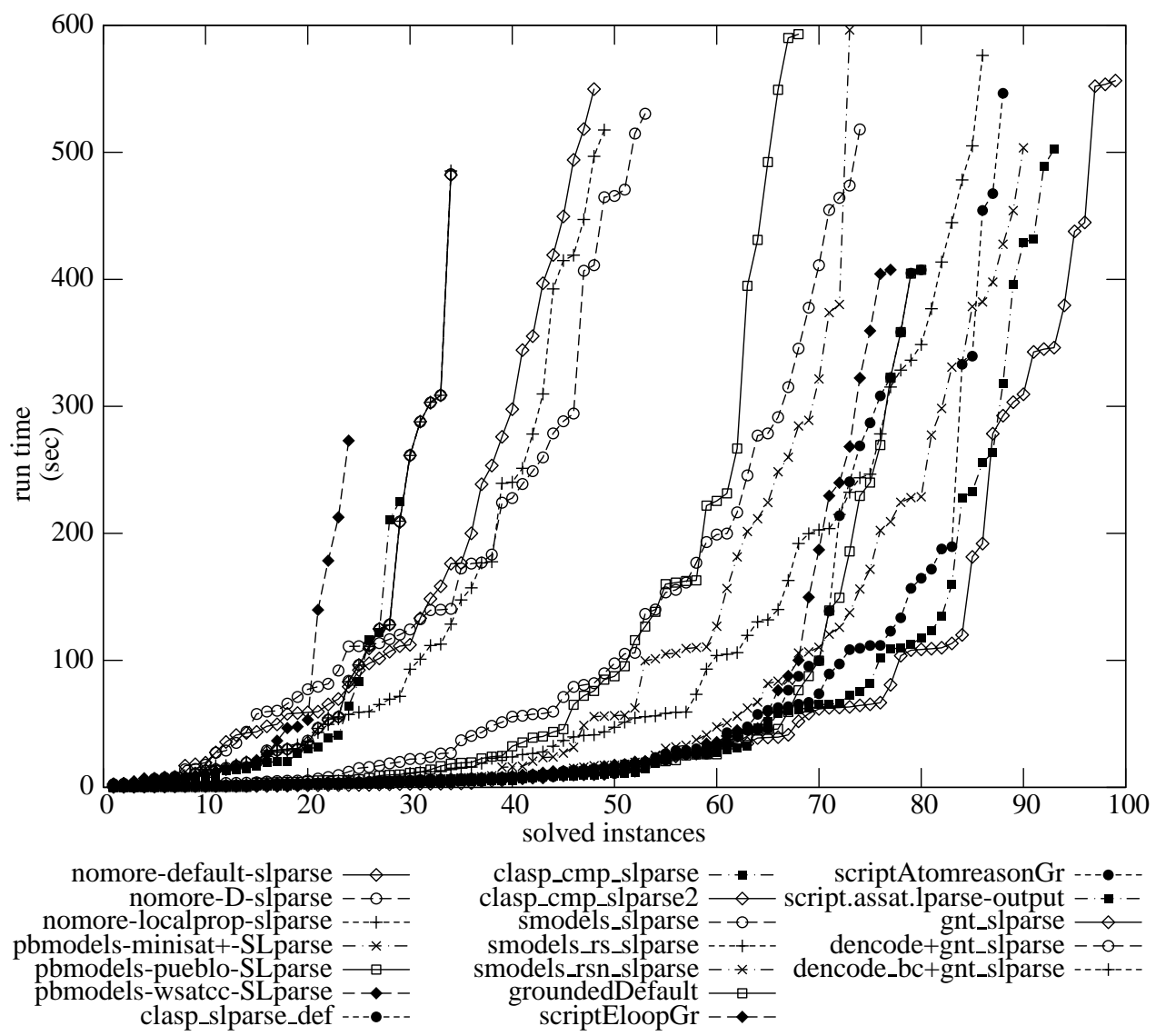

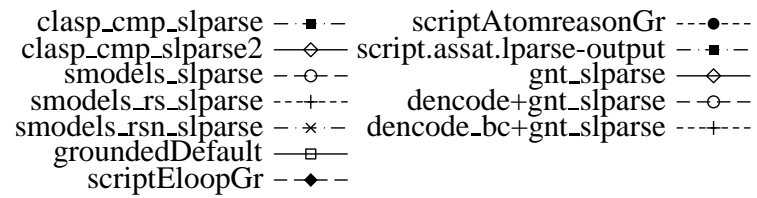

Fig. 4. Chart of the SLparse competition.

\section{FIRST Place WinNer clasp \\ SECOND PLACE WINNER pbmodels \\ THIRD PLACE WINNER smodels}

The detailed placement of call scripts is given in Table 9. Table 10 gives statistics about the benchmark classes used in the SLparse competition. The performance of all participating call scripts is charted in Figure 4.

\section{Discussion}

This First Answer Set Programming System Competition offers many interesting lessons stemming from running diverse solvers on multifaceted benchmark instances. Some of the lessons may have general implications on the future developments in ASP.

First, the experiences gained from the effort to design the competition clearly point out that the lack of well-defined input, intermediate, and output languages is a major 
problem. In some cases, it forced the competition team to resort to "ad hoc" solutions. Further, there is no standard core ASP language covering programs with aggregates, which makes it difficult to design a single and fair field for all systems to compete. No standard way in which errors are signaled and no consensus on how to deal with incomplete solvers are somewhat less critical but also important issues. Benchmark selection is a major problem. The way benchmarks and their instances are chosen may have a significant impact on the results in view of diverging performances of solvers and different degrees of difficulty among the instances of benchmark classes. Sometimes even grounding problem encodings on problem instances to produce ground programs on which solvers were to compete was a major hurdle (see below).

This first edition of the competition focused on the performance of solvers on ground programs, which is certainly important. However, the roots of the ASP approach are in declarative programming and knowledge representation. For both areas, modeling knowledge domains and problems that arise in them is of major concern (this is especially the case for knowledge representation). By developing the MGS category, we tried to create a platform where ASP systems could be differentiated from the perspective of their modeling functionality. However, only one group chose to develop programs specialized to their system (hence, this group and their system are the welldeserved winner). All other groups relied on default encodings. It is critical that a better venue for testing modeling capabilities is provided for future competitions.

Further, not only modeling support and the performance of solvers determine the quality of an ASP system. Grounding is an essential part of the process too and, in some cases, it is precisely where the bottleneck lies. The MGS category was the only category that took both the grounding time and the solving time into account. It is important to stress more the role of grounding in future competitions.

There will be future competitions building on the experiences of this one. Their success and their impact on the field will depend on continued broad community participation in fine-tuning and expanding the present format. In this respect, the First Answer Set Programming System Competition should encourage the further progress in the development of ASP systems and applications, similar to competitions in related areas, such as SATisfiability, Quantified Boolean Formulas, and Pseudo-Boolean constraints.

\section{Acknowledgments}

This project would not have been possible without strong and broad support from the whole ASP community. We are grateful to all friends and colleagues who contributed ideas on the contest format, submitted benchmark problems, and provided continued encouragement. Most of all, we want to thank all the competitors. Without you, there would have been no competition.

Mirosław Truszczyński acknowledges the support of NSF grant IIS-0325063 and KSEF grant 1036-RDE-008.

\section{References}

1. Niemelä, I.: Logic programming with stable model semantics as a constraint programming paradigm. Annals of Mathematics and Artificial Intelligence 25(3-4) (1999) 241-273 
2. Marek, V., Truszczyński, M.: Stable models and an alternative logic programming paradigm. In Apt, K., Marek, W., Truszczyński, M., Warren, D., eds.: The Logic Programming Paradigm: a 25-Year Perspective. Springer (1999) 375-398

3. Gelfond, M., Leone, N.: Logic programming and knowledge representation - the A-prolog perspective. Artificial Intelligence 138(1-2) (2002) 3-38

4. Baral, C.: Knowledge Representation, Reasoning and Declarative Problem Solving. Cambridge University Press (2003)

5. Colmerauer, A., Kanoui, H., Pasero, R., Roussel, P.: Un systeme de communication hommemachine en Francais. Technical report, University of Marseille (1973)

6. Kowalski, R.: Predicate logic as a programming language. In Rosenfeld, J., ed.: Proceedings of the Congress of the International Federation for Information Processing, North Holland (1974) 569-574

7. McCarthy, J.: Circumscription - a form of nonmonotonic reasoning. Artificial Intelligence 13(1-2) (1980) 27-39

8. Reiter, R.: A logic for default reasoning. Artificial Intelligence 13(1-2) (1980) 81-132

9. Gelfond, M., Lifschitz, V.: The stable model semantics for logic programming. In Kowalski, R., Bowen, K., eds.: Proceedings of the International Conference on Logic Programming, MIT Press (1988) 1070-1080

10. Gelfond, M., Lifschitz, V.: Classical negation in logic programs and disjunctive databases. New Generation Computing 9(3-4) (1991) 365-385

11. Marek, W., Truszczyński, M.: Stable semantics for logic programs and default theories. In Lusk, E., Overbeek, R., eds.: Proceedings of the North American Conference on Logic Programming, MIT Press (1989) 243-256

12. Bidoit, N., Froidevaux, C.: Negation by default and unstratifiable logic programs. Theoretical Computer Science 78(1) (1991) 85-112

13. Eiter, T., Leone, N., Mateis, C., Pfeifer, G., Scarcello, F.: A deductive system for nonmonotonic reasoning. In Dix, J., Furbach, U., Nerode, A., eds.: Proceedings of the International Conference on Logic Programming and Nonmonotonic Reasoning, Springer (1997) 364-375

14. Niemelä, I., Simons, P.: Smodels — an implementation of the stable model and well-founded semantics for normal logic programs. In Dix, J., Furbach, U., Nerode, A., eds.: Proceedings of the International Conference on Logic Programming and Nonmonotonic Reasoning, Springer (1997) 420-429

15. Borchert, P., Anger, C., Schaub, T., Truszczyński, M.: Towards systematic benchmarking in answer set programming: The Dagstuhl initiative. In Lifschitz, V., Niemelä, I., eds.: Proceedings of the International Conference on Logic Programming and Nonmonotonic Reasoning, Springer (2004) 3-7

16. Le Berre, D., Simon, L., eds.: Special Volume on the SAT 2005 Competitions and Evaluations. Journal on Satisfiability, Boolean Modeling and Computation 2(1-4) (2006)

17. (http://asparagus.cs.uni-potsdam.de/contest)

18. (http://asparagus.cs.uni-potsdam.de)

19. Leone, N., Pfeifer, G., Faber, W., Eiter, T., Gottlob, G., Perri, S., Scarcello, F.: The DLV system for knowledge representation and reasoning. ACM Transactions on Computational Logic 7(3) (2006) 499-562

20. Syrjänen, T.: Lparse 1.0 user's manual. (http://www.tcs.hut.fi/Software/smodels/lparse.ps.gz) 